\title{
$\begin{array}{ll}\text { Research Square } & \text { Preprints are preliminary reports that have not undergone peer review. } \\ \text { They should not be considered conclusive, used to inform clinical practice, }\end{array}$
}

\section{Development and Validation of Self-screening Tool for Nutrition Risk in Patients With Gastric Cancer After Gastrectomy: A STUDY PROTOCOL}

\author{
Zhiming Chen \\ Jilin University \\ Haichi Yu \\ Jilin University Second Hospital \\ Hua Yuan \\ Jilin University \\ Jia Wang \\ Jilin University \\ Qiuchen Wang \\ Jilin University \\ Mingyue Zhu \\ Jilin University \\ jiannan Yao \\ Jilin University \\ Xiuying Zhang ( $\sim$ z_xy@jlu.edu.cn ) \\ Jilin University \\ Hui Xue \\ Jilin University
}

Study protocol

Keywords: Gastric Cancer, Self-screening, Nutritional screening, Questionnaire, Protocol

Posted Date: November 29th, 2021

DOI: https://doi.org/10.21203/rs.3.rs-1065435/v1

License: @ (1) This work is licensed under a Creative Commons Attribution 4.0 International License. Read Full License 


\section{Abstract}

Background: The incidence of malnutrition in patients with gastric cancer after surgery is $59 \%$. The main reason for the high incidence of malnutrition is untimely nutrition screening and low compliance with nutrition treatment. In order to enable home patients to know their nutritional status in time, we have developed and validated nutritional risk screening tools for patients with gastric cancer to help patients' at home find nutritional risks in time and seek medical help. This article introduces the development and verification methods and statistical methods of the tool.

Methods: The development of self-nutrition risk screening tool for patients with gastric cancer after gastrectomy (SNRSGC) is divided into four parts $\llbracket$ Steps 1 Identification of a potential referred nutritional risk screening; Steps2Item generation and scoring are selected through literature review methods to screen sensitive indicators that can reflect the nutritional characteristics of patients after gastric

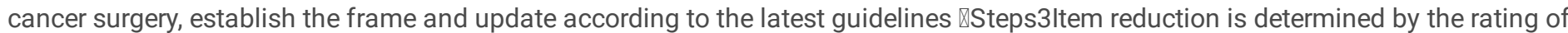
SNRSGC items by an expert panel and piloting method to determine the final item; Steps 4 In the validation stage, we conducted research design based on the Consensus-based Standards for the selection of health Measurement Instruments checklist to evaluate the content validity, structure validity, interpretability, and retest validity of SNRSGC.

Discussion: SNRSGC is the first screening tool specifically to predict nutrition risk for stay-at-home postoperative patients with gastric cancer.SNRSGC may provide action guidelines and knowledge guidance for patients with gastric cancer at home.

Trial registration: Identifier on Chinese Clinical Trials Registry : ChiCTR2100041809, registered January 06, 2021.

\section{Introduction}

Gastric cancer is the fifth most common cancer and the third leading cause of cancer death in the world. According to cancer statistics published by the World Health Organization in February 2021, there are 479,000 new cases of gastric cancer in China each year, accounting for $43.9 \%$ of the global new cases of gastric cancer[1].

The incidence of postoperative malnutrition in patients with gastric cancer was 30\%-59\% and lasts for One year after operation.[2-5] The malnutrition in patients with gastric cancer not only increases the burden of the medical system due to the high readmission rate and long hospital stay[6], but also reduces the survival time of patients due to high postoperative complications and low quality of life[3,7,8]. Nutritional therapy is the preferred treatment for malnutrition [9-11]. Nutritional risk screening is both the starting point of the nutrition therapy and can guide the nutrition therapy plan[9,12].In addition, the European nutrition guidelines suggest that cancer patients should regularly carry out nutritional risk screening[10]. At present, Nutritional risk screening mainly relies on nutritional risk screening tools, including Nutritional Risk Screening 2002 (NRS2002) [13], Mini Nutritional Assessment (MNA), Malnutrition Universal Screening Tool (MUST), etc. The screening tools need to be performed by medical workers due to professional words and calculation formulas in their items [13-15]. This need for specialization bring the following challenges: 1 The regular nutritional screening will undoubtedly further increase the workload and/or number of medical workers[16,17]; 2 Patients did not timely seek treatment not to get screening effectively because they did not realize nutritional risks related problems. Therefore『untimely and regularly nutritional risk screening is a big challenge in the process of nutrition management for discharged patients with gastric cancer.

Self-screening may provide a regular and effective nutritional risk screening way to promote medical treatment, prevent malnutrition and start or revise nutritional treatment programs for discharged patients with gastric cancer [18-20]. Research confirmed self-nutrition risk screening for gastrointestinal outpatients and cancer outpatients using MUST and MST has been proved to be feasible and reliable[21,22]. However, Existing nutritional risk screening tools including NRS2002, MNA, MUST, etc. are not suitable for self-nutrition risk screening for gastric cancer patients. Frist, they were mainly developed with clinical patients, the elderly and cancer patients as the sample population[13-15] . In patients with gastric cancer after surgery, the screening results of different tools are quite different and the accuracy is low $(20.6 \%-63.2 \%)[23,24]$. This may be related to the fact that these nutritional risk screening tools were not developed for the sample population of patients after gastric cancer surgery, and did not contain their specific nutritional indicators. For example邓MUST only include weight loss but not intake reduction_that is an important factor for malnutrition in patients with gastric cancer[23,25].Only NRS2002 contains the influence of surgical indicators on the nutritional status of patients. According to their scoring criteria, the effect of surgery on nutritional status in a short time after operation may be underestimated [26,27]. Second, most of the tools include professional words and calculation formulas that patients can't understand囚which has hampered its use in community and family. The development of self-nutrition risk screening tools can enable patients to find early lesions in time, increase treatment compliance and 
reduce the work pressure of health care workers[18-20]. Therefore, developing an instrument that provides a valid assessment of selfnutritional risk screening and easy-to-understand for patients is crucial.

The aim of this protocol is to describe the method and analysis plan for the development and validation of a self-nutritional risk screening tool for patients with gastric cancer after gastrectomy that is suitable for preventing malnutrition and starting or revising nutritional treatment programs.

\section{Methods}

The Consensus-based Standards for the selection of health Measurement Instruments (COSMIN) checklist for developing and evaluating the self-nutrition risk screening tool for patients with gastric cancer after gastrectomy (SNRSGC) included the following: steps 1 identification of a potential referred nutritional risk screening tool. steps 2 items and scores generation, steps 3 item reduction and steps 4_determination of the validity, reliability and responsiveness. Steps 1 and 2 involved developing a preliminary version of the questionnaire, which is described in the methods section. Step 3 involved testing the individual items and subscales of the preliminary version by analyzing patient responses. Based upon these analyses, a final version of the questionnaire was decided upon. Step 4 involved testing the final version of the questionnaire for validity, reliability and responsiveness. A flowchart of the complete study process is shown in figure 1.

\section{1ldentification of a potential referred nutritional risk screening tool}

At present, research shows that the accuracy of screening tools can be improved by revising the entries of existing screening tools[28,29]. The goal of this instrument is to make postoperative patients with gastric cancer themselves evaluate nutritional risk screening. NRS2002 as convenience for application and less time consumption, has been widely used in clinical practice and recommended by many guidelines for nutritional assessment of patients undergoing abdominal surgery[30,31]. Moreover, compared with other nutritional risk screening tools such as MAN and MUST, it included the risk factors related to the patients' nutrition such as surgery and chemotherapy. In addition, NRS2002 had a strong capacity to identify patients with cachexia (AUC=0.916)[25]. NRS2002 were significantly associated with overall survival and nutritional measurement tools (BMI and anthropometric measurements) at six months after surgery[25,32]. Therefore, NRS2002 was selected as a potential referred nutritional risk screening tool for the development of the SNRSGC.

\section{2ltems and scores generation}

In the item and scores generation phase, the following steps was operated:

(1) adjusting to establish the frame of SNRSGC according to the original study about NRS2002 [13].

The original developers of the NRS2002 were contacted to ask for permission to adapt the NRS2002 to develop SNRSGC suitable to patients. The main of some items in the NRS2002 did not understoode by the patients because the users of it was expected to be health care personnel such as calculation of BMI, daily intake and body energy demand. Therefore, the items will be revised or deleted.

Moreover, some items related to the situations did not occur in the discharged patient will also be deleted such as intensive care patients, bone marrow transplantation and severe pneumonia. Though the some items in it had to been deleted, the basic framework of the NRS2002 "undernutrition囚disease severity \age" was retained.

The determination of the scores in the items refers to the research hypothesis of NRS2002 that patients can benefit from nutritional support. For example, scoring standard: "Score=1, Protein requirement is increased, but in most case it can be met by oral diet or supplements. Score=2, Protein requirement is substantially increased but it can be met, although artificial feeding is required in many cases. Score=3, The protein requirement increases, so that in most cases, even artificial feed cannot be met, but protein breakdown and $\mathrm{N}$ loss can be attenuated significantly."

(2) conducting a systematic review of the literature.

Most scholars suggest selecting an appropriate nutritional risk screening tool for different groups of people, or revising the scale according to the characteristics of different diseases [14]. The systematic review identified existing the specific nutritional indicators of postoperative patients with gastric cancer that were expected to improve the accuracy of screening. The factors that affect the nutritional status of postoperative patients with gastric cancer was chosen as a template for the development of the SNRSGC's items. A literature search was conducted to obtain the factors using electronic databases Medline, PubMed, China National Knowledge Infrastructure and 
web of science. The search keywords included: nutrition /malnutrition /gastric cancer /stomach Neoplasms, and a manual review of the references in the selected studies was performed to identify further publications. The determination of the inclusion and exclusion criteria of the studies refers to the research hypothesis of NRS2002 that patients can benefit from nutritional supplement. The inclusion and exclusion criteria of the studies was showed in the table 1.

(3) Updated and refer to the cut-off value according to GLIM.

As malnutrition assessment and diagnosis methods are constantly updated, the development of tools should be revised in conjunction with the latest nutrition guidelines. In addition, some nutritional index cut-off values in NRS2002 are not applicable to Chinese patients, so it is necessary to change the items in it[33] . According to Global Leadership Initiative on Malnutrition (GLIM)[12], easy to measure and necessary nutrition measurement indexes are added in SNRSGC and refer to the cut-off value of nutrition indexes for Asians given therein.

\subsection{Item reduction process}

Rating of SNRSGC items by an expert panel

The second step involved expert enquiry in the field. In order to allow multi-professional cooperation and comprehensive evaluation of items, the complete item pool will be evaluated by 15 experts with knowledge of nursing, nutrition and medical of postoperative patients with gastric cancer. The experts involved in the study (1) who have more than 10 years working experience in this field (2) who with intermediate title or above (3) who with bachelor degree or above. This process of designing uses questionnaire literature methodology. Experts were required to evaluate each item and scores on the consultation list according to the importance, the basis and familiarity and put forward comments and proposal for relevant indicators. Experts will be asked to rate the relevance of each item on a Likert scale: 1 (not relevant); 2 (item needs some revision); 3 (relevant but needs minor revision); and 4 (very relevant) as well as provide an explanation for their decision and suggestions on any missing items. Respondents could participate in a WeChat or telephone call with the lead author to provide further feedback, if desired. All written and verbal feedback was analyzed, and minor wording revisions were made to produce the final tool. The purpose of the rating of SNRSGC items by an expert panel was to identify relevant items that were missing and to improve the readability and comprehension of the questionnaire.

Based upon the first and second administration of the preliminary SNRSGC version, item reduction was performed using the following strategy, which incorporated both expert and patient's opinions. The item-level content validity index (I-CVI) was calculated for each principle and item (number of respondents giving a rating of either 3 or 4, divided by the total number of respondents)[34]. One weakness of CVI is its failure to adjust for chance agreement. We solved this by translating item-level CVIs (I-CVIs) into values of a modified kappa statistic. Denise F. Polit et al. believe that items with an I-CVI of 0.78 or higher can be considered as evidence of good content validity[34]. Items with I-CVI less than 0.78 should be corrected, and items less than 0.5 should be deleted[34]. If the experts' ratings of item relevance are quite different, we will do second-round panel and calculate S-CVI.

Piloting

The final step in the item generation process was to interview postoperative patients with gastric cancer and their families individually. The final version was made into a WeChat mini program and distributed to patients or family members. The postoperative patients with gastric cancer and their families in the outpatient clinic were randomly selected and asked to assess the difficulty of each item in the SNRSGC questionnaire, as very easy, easy, not easy-not hard, hard, or very hard. Inclusion criteria: patients were those: Age > 18 years old; the postoperative patients with gastric cancer; patients and/or patients' families with reading ability, clear consciousness and normal expression ability and using mobile software. Exclusion criteria: Patients with other malignant tumors; Patients with metabolic diseases; Palliative surgery patients. The purpose of the patients and their families interviews was to identify relevant items that were missing, to improve the readability and comprehension of the questionnaire and evaluate the patient cognitive burden of screening methods.

The patient's assessment of the difficulty of SNRSGC items is expressed as a percentage. When the number of people who choose "hard" exceeds $50 \%$, the item should be revised.

\subsection{To evaluate the validity, reliability and patient acceptability of SNRSGC.}

Methodological testing and evaluation of measurement qualities of SNRSGC using the COSMIN checklist 
We evaluated the final SNRSGC measurement properties according to the_consensus -based Standards for the selection of health Measurements Instruments (COSMIN) guidelines. The purpose of the COSMIN checklist is to evaluate the methodological quality of studies concerning measurement properties of health-related patient-reported outcomes (HR-PROs) instruments.[35,36]

Participants

Participant were recruited from the inpatient and outpatient department of gastrointestinal surgery in a large tertiary hospital in China. Patients eligible for inclusion were those: Age > 18 years old; The patients with gastric cancer confirmed by histopathological biopsy were operated on; Be able to read the scale; Clear consciousness and normal expression ability; Able to use mobile software. Exclusion criteria; Patients with other malignant tumors; Patients with metabolic diseases; Palliative surgery patients; The patients who could not eat by mouth after operation; Refused to participate in this study through communication.

Caregiver eligible for inclusion were those who provided care for the gastric cancer surgery patients older than 18 years in the past three months and were able to read the scale; Clear consciousness and normal expression ability; Able to use mobile software.

Sample size calculation: According to the minimum sample size of diagnostic experiment, the minimum sample size is 151[32,37]. The minimum sample size for test-retest is 50[38]. In order to ensure the validity of the statistical results, we adopt the stratified sampling method. Stratified according to the postoperative time, divided into three months after surgery and more than three months after surgery, the number of people in each group is greater than 50[36].

According to the usage of NRS2002 and SNRSGC, data collection and inclusion and exclusion criteria, a training slide was made for researchers to conduct training. The researcher provided the questionnaire to the patients in the form of a mini program and collected the data. When the patients filled in the questionnaire, the researcher evaluated the NRS2002 score of the patients.

Internal consistency

Internal consistency or item homogeneity is often used for estimating intra-scale reliability in terms of the item variances and covariances derived from a single occasion of measurement. A principal component factor analysis was performed on the individual subscales to assess their structural validity. Cronbach's a was calculated per subscale and a score above 0.70 was taken as an indication of sufficient homogeneity of the items in the subscale[39,40].

Construct validity

Construct validity refers to the extent to which scores on a particular instrument relate to other measures. This construct validity was determined by cross-sectional comparison of the questionnaires when first administered._We conducted exploratory factor analysis to explore the unidimensionality of SNRSGC item. Items were considered to load on a factor if the factor loading was 0.32 or greater[41]. The tool developed in this research aims to distinguish whether patients have nutritional risks and whether patients can benefit from nutritional supplements[42]. Since there is no gold standard for health self-reporting, we cannot compare NRS2002 as a gold standard with SNRSGC. The expert group of COSMIN checklist recommends that it be regarded as structural validity[36]. Therefore, the area under the receiver operating characteristic (ROC) curve, sensitivity and specificity were determined to investigate the concurrent validity of the SNRSGC considering the original NRS2002 as the reference method. Area under the receiver operating characteristic curve (AUC) $>0.7$ is considered adequate[38].

Test-retest reliability

Test-retest reliability refers to the similarity of the scores of the same patient after repeated measurement over a period of time. Testretest reliability is divided into two parts: agreement and reliability. The parameter of agreement in this study uses the description of Bland and Altman. Their limits of agreement equal the mean change in scores of repeated measurements (mean change) $\pm 1.96 \times$ standard deviation of these changes (SD change). The limits of agreement are often reported because they are easily interpretable. Note that SD change equals $\sqrt{ } 2 \times$ SEM consistency. SEM equals the square root of the error variance of an ANOVA analysis. We used intraclass correlation coefficient (ICC) to evaluate reliability, with Kappa values $\geq 0.7$ considered adequate.

Patients reporting scores as 'unchanged' were considered stable and included in test-retest reliability analysis. Since gastric cancer patients have large changes in body weight and nutritional status within three months after surgery[26,43]邓they are not suitable for 
testing the reliability of test-retest reliability; Therefore, we tested the test-retest reliability of patients three months after operation. The interval between the two surveys was one week.

Interpretability

Interpretability is the degree to which one can assign qualitative meaning to an instrument's quantitative scores or change in scores.

Interpretability refers to the degree of qualitative significance of the quantitative score of SNRSGC. Interpretability includes the distribution of total scores and change scores in the study sample and in relevant subgroups, floor and ceiling effects, estimates of minimal important change (MIC) and/or minimal important difference (MID)[44]. If the highest or lowest score exceeds 15\%, the validity of the tool content is considered limited. Patients with the lowest or highest scores cannot be distinguished from each other, reducing reliability.

Acceptability

The acceptability of the SNRSGC was assessed by the patient with an additional questionnaire. The patient categorically assessed the difficulty of the SNRSGC questionnaire, as very easy, easy, not easy-not hard, hard, or very hard. Furthermore, the patient assessed the time spent on the SNRSGC categorically as $<5,5-10,10-15$, or $>15 \mathrm{~min}$.

Data collection included patient's SNRSGC score, time of SNRSGC, NRS2002 score, difficulty of questionnaire, tumor stage, filling person's educational background, postoperative time and so on.

\section{Discussion}

We have described the methods and statistical analysis plan to develop and validate a SNRSGC for postoperative patients with gastric cancer. To our knowledge, SNRSGC is the first screening tool specifically to predict nutrition risk for stay-at-home postoperative patients with gastric cancer. Furthermore, this is one of the first studies following the full COSMIN checklist, the recent international consensus process involving leading experts in the development and testing of PRO questionnaires [35,36]. In addition, in this study, WeChat applet, a popular, convenient,_mobile technology among patients, was considered as a screening approach to increase the acceptability of selfscreening.

SNRSGC is a distinguishing tool for the nutritional risk screening of gastric cancer patients at home. According to the guidance of the basic elements of questionnaire development and design[45], this study conducted a systematic literature review based on the development hypothesis of NRS2002, so that the screening tool contains specific indicators that can reflect the nutritional status of patients with gastric cancer after surgery, and ensure that the patients screened by the tool can benefit from nutritional supplements. Expert questionnaire surveys and reference to GLIM ensure the comprehensiveness of the items and prepare for the later selection of effective indicators. Patient surveys ensure the readability and availability of entries.

NRS2002 is recommended by various nutrition guidelines in China for nutritional screening of hospitalized patients and community populations, and is widely used by health care personnel. The development of NRS2002 as a template helps to establish a consensus on nutrition assessment between nurses and patients or between doctors and patients. Compared with other scales, it also includes the effects of surgery and chemotherapy on the nutritional status of patients, and is closer to the characteristics of the course of gastric cancer patients. And it has the characteristics of simple entry and convenient use, simple and objective indicators help to improve the convenience and accuracy of patient self-screening.

COSMIN was developed in an international Delphi study to assess the methodological quality of research on the measurement characteristics of health-related patient-reported outcomes (HR-PROs)[36]. We conducted an experimental design for the reliability and validity of SNRSGC according to the items and requirements of the COSMIN evaluation. On the one hand, COSMIN is designed to evaluate the HR-PROs, which fits the research purpose of this study. On the other hand, COSMIN is a systematic evaluation form, which can comprehensively evaluate the performance of SNRSGC in all aspects, and can ensure the rigor and effectiveness of the development and inspection of SNRSGC[46].

In terms of study design, we did not design the effect of SNRSGC at home after gastric cancer surgery. SNRSGC is a discriminative questionnaire, so our protocol does not evaluate the responsiveness of SNRSGC. In future studies, we will continue to further evaluate the effect of SNRSGC in patients with gastric cancer at home after gastrectomy. Because the self-management of cancer patients has achieved good results in weight maintenance[47,48]. However, 83\% of patients with gastric cancer had malnutrition 6 months after 
operation, and weight loss lasted for two years after operation, which means that patients with gastric cancer may have persistent malnutrition and poor self-management ability of nutrition[5,43]. The use of SNRSGC may have a positive impact on the nutritional management of gastric cancer patients and improve the patient's outcome.

In previous studies, we found that the electronic version of nutritional risk screening can provide great convenience for patients' autonomous screening and is easier to be accepted by patients[21]. Therefore, we choose a WeChat applet that is highly acceptable to patients to design SNRGC to enable the tools are simple and easy to obtain. Regular screening can improve patients' nutritional awareness [20], allow patients to understand their physical condition, and promote patients to take the initiative to seek medical treatment[18]. Nutrition self-screening tools can also increase patient compliance with treatment and give patients practical guidance[19,20]. Achieve timely detection of nutritional risks, early start of nutritional procedures, increase compliance with nutritional therapy, improve nutritional status, extend patient survival, and improve patient quality of life.

Limitations in the use of tools. Because we use the form of online questionnaire for self-screening, although it is easy to obtain, some elderly people who do not use mobile phone software are difficult to conduct self-screening, and they may have worse nutritional status. Moreover, due to the function setting of the software, patients must fill in all the contents to give the results, which greatly reduces the recovery rate of the questionnaire, but increases the efficiency. Limitations of the study design. Although we use patients three months after operation for retest, the nutritional status of patients is still unstable due to chemotherapy and some long-term postoperative complications, which may affect the test-retest reliability.

\section{Conclusion}

This protocol overviews the design of the SNRSGC development and verification study. The results of this study will be used for clinical and research purposes.

\section{Declarations}

\section{Acknowledgments}

We gratefully acknowledge the expert assistance of Haiyan Hu and Qing Zhang.

\section{Ethics approval and consent to participate}

The original study that we plan to analyze using the methods outlined in this protocol has been ethically approved by the Ethics Committee of the School of Nursing, Jilin University.

\section{Consent for publication}

Not Applicable.

\section{Availability of data and materials}

Not applicable

\section{Competing of Interest}

The authors declare no conflict of interest. The study has not been peer-reviewed.

\section{Funding:}

This work was supported by the National Natural Science Foundation of China [grant numbers 31800895].The funders had no role in study design, data collection and analysis, decision to publish, or preparation of the manuscript.

\section{Authors' Contributions}

All the authors have participated in the research work and assumed public responsibility for the research paper, including: significant contributions to the relevant concepts and design of this research,revising the important knowledge contents of the paper repeatedly;approving the final version and agreeing to be responsible for all aspects of the work. Zhiming Chen and Haichi Liu contributed equally to this work. 


\section{References}

1. International Agency for Research on cancer. Availabe online: https://gco.iarc.fr/today (accessed on

2. Li, Q.; Zhang, X.; Tang, M.; Song, M.; Zhang, Q.; Zhang, K.; Ruan, G.; Zhang, X.; Ge, Y.; Yang, M., et al. Different muscle mass indices of the Global Leadership Initiative on Malnutrition in diagnosing malnutrition and predicting survival of patients with gastric cancer. Nutrition (Burbank, Los Angeles County, Calif.) 2021, 89, 111286, doi:10.1016/j.nut.2021.111286.

3. Fujiya, K.; Kawamura, T.; Omae, K.; Makuuchi, R.; Irino, T.; Tokunaga, M.; Tanizawa, Y.; Bando, E.; Terashima, M. Impact of Malnutrition After Gastrectomy for Gastric Cancer on Long-Term Survival. Annals of surgical oncology 2018, 25, 974-983, doi:10.1245/s10434018-6342-8.

4. Seo, S.H.; Kim, S.E.; Kang, Y.K.; Ryoo, B.Y.; Ryu, M.H.; Jeong, J.H.; Kang, S.S.; Yang, M.; Lee, J.E.; Sung, M.K. Association of nutritional status-related indices and chemotherapy-induced adverse events in gastric cancer patients. BMC cancer 2016, 16, 900, doi:10.1186/s12885-016-2934-5.

5. Davis, J.L.; Selby, L.V.; Chou, J.F.; Schattner, M.; Ilson, D.H.; Capanu, M.; Brennan, M.F.; Coit, D.G.; Strong, V.E. Patterns and Predictors of Weight Loss After Gastrectomy for Cancer. Annals of surgical oncology 2016, 23, 1639-1645, doi:10.1245/s10434-015-5065-3.

6. Pimiento, J.M.; Evans, D.C.; Tyler, R.; Barrocas, A.; Hernandez, B.; Araujo-Torres, K.; Guenter, P. Value of nutrition support therapy in patients with gastrointestinal malignancies: a narrative review and health economic analysis of impact on clinical outcomes in the United States. J Gastrointest Oncol 2021, 12, 864-873, doi:10.21037/jgo-20-326.

7. Fukuda, Y.; Yamamoto, K.; Hirao, M.; Nishikawa, K.; Maeda, S.; Haraguchi, N.; Miyake, M.; Hama, N.; Miyamoto, A.; Ikeda, M., et al. Prevalence of Malnutrition Among Gastric Cancer Patients Undergoing Gastrectomy and Optimal Preoperative Nutritional Support for Preventing Surgical Site Infections. Annals of surgical oncology 2015, 22 Supp/ 3, S778-785, doi:10.1245/s10434-015-4820-9.

8. Kaźmierczak-Siedlecka, K.; Skonieczna-Żydecka, K.; Folwarski, M.; Ruszkowski, J.; Świerblewski, M.; Makarewicz, W. Influence of malnutrition stage according to GLIM 2019 criteria and SGA on the quality of life of patients with advanced cancer. Nutricion hospitalaria 2020, 37, 1179-1185, doi:10.20960/nh.03185.

9. Deftereos, I.; Kiss, N.; Isenring, E.; Carter, V.M.; Yeung, J.M. A systematic review of the effect of preoperative nutrition support on nutritional status and treatment outcomes in upper gastrointestinal cancer resection. European journal of surgical oncology: the journal of the European Society of Surgical Oncology and the British Association of Surgical Oncology 2020, 46, 1423-1434, doi:10.1016/j.ejso.2020.04.008.

10. Muscaritoli, M.; Arends, J.; Bachmann, P.; Baracos, V.; Barthelemy, N.; Bertz, H.; Bozzetti, F.; Hütterer, E.; Isenring, E.; Kaasa, S., et al. ESPEN practical guideline: Clinical Nutrition in cancer. Clinical nutrition (Edinburgh, Scotland) 2021, 40, 2898-2913, doi:10.1016/j.clnu.2021.02.005.

11. Lobo, D.N.; Gianotti, L.; Adiamah, A.; Barazzoni, R.; Deutz, N.E.P.; Dhatariya, K.; Greenhaff, P.L.; Hiesmayr, M.; Hjort Jakobsen, D.; Klek, S., et al. Perioperative nutrition: Recommendations from the ESPEN expert group. Clinical nutrition (Edinburgh, Scotland) 2020, 39, 3211-3227, doi:10.1016/j.clnu.2020.03.038.

12. Cederholm, T.; Jensen, G.L.; Correia, M.; Gonzalez, M.C.; Fukushima, R.; Higashiguchi, T.; Baptista, G.; Barazzoni, R.; Blaauw, R.; Coats, A., et al. GLIM criteria for the diagnosis of malnutrition - A consensus report from the global clinical nutrition community. Clinical nutrition (Edinburgh, Scotland) 2019, 38, 1-9, doi:10.1016/j.clnu.2018.08.002.

13. Kondrup, J.; Rasmussen, H.H.; Hamberg, O.; Stanga, Z. Nutritional risk screening (NRS 2002): a new method based on an analysis of controlled clinical trials. Clinical nutrition (Edinburgh, Scotland) 2003, 22, 321-336, doi:10.1016/s0261-5614(02)00214-5.

14. van Bokhorst-de van der Schueren, M.A.; Guaitoli, P.R.; Jansma, E.P.; de Vet, H.C. Nutrition screening tools: does one size fit all? A systematic review of screening tools for the hospital setting. Clinical nutrition (Edinburgh, Scotland) 2014, 33, 39-58, doi:10.1016/j.clnu.2013.04.008.

15. Rubenstein, L.Z.; Harker, J.O.; Salvà, A.; Guigoz, Y.; Vellas, B. Screening for undernutrition in geriatric practice: developing the shortform mini-nutritional assessment (MNA-SF). J Gerontol A Biol Sci Med Sci 2001, 56, M366-372, doi:10.1093/gerona/56.6.m366.

16. Lawler, J.; Leary, A.; Lofton, L.; Bushe, D. Perceptions of the cancer care left undone in primary and community services: A mixed methods evaluation. Health Soc Care Community 2020, 28, 2117-2124, doi:10.1111/hsc.13022.

17. Arends, J.; Bachmann, P.; Baracos, V.; Barthelemy, N.; Bertz, H.; Bozzetti, F.; Fearon, K.; Hütterer, E.; Isenring, E.; Kaasa, S., et al. ESPEN guidelines on nutrition in cancer patients. Clinical nutrition (Edinburgh, Scotland) 2017, 36, 11-48, doi:10.1016/j.clnu.2016.07.015.

18. Ghani, W.M.N.; Razak, I.A.; Doss, J.G.; Ramanathan, A.; Tahir, Z.; Ridzuan, N.A.; Edgar, S.; Zain, R.B. Mouth self-examination as a screening tool for oral potentially malignant disorders among a high-risk Indigenous population. J Public Health Dent 2019, 79, 222-

Page $8 / 11$ 
230, doi:10.1111/jphd.12313.

19. Vernooij, R.W.; Willson, M.; Gagliardi, A.R. Characterizing patient-oriented tools that could be packaged with guidelines to promote self-management and guideline adoption: a meta-review. Implementation science: IS 2016, 11, 52, doi:10.1186/s13012-016-0419-1.

20. Jager-Wittenaar, H.; de Bats, H.F.; Welink-Lamberts, B.J.; Gort-van Dijk, D.; van der Laan, B.; Ottery, F.D.; Roodenburg, J.L.N. SelfCompletion of the Patient-Generated Subjective Global Assessment Short Form Is Feasible and Is Associated With Increased Awareness on Malnutrition Risk in Patients With Head and Neck Cancer. Nutrition in clinical practice: official publication of the American Society for Parenteral and Enteral Nutrition 2020, 35, 353-362, doi:10.1002/ncp.10313.

21. Cawood, A.L.; Walters, E.R.; Sharp, S.K.E.; Elia, M.; Stratton, R.J. 'Self-screening' for malnutrition with an electronic version of the Malnutrition Universal Screening Tool ('MUST') in hospital outpatients: concurrent validity, preference and ease of use. The British journal of nutrition 2018, 120, 528-536, doi:10.1017/s000711451800185x.

22. Di Bella, A.; Croisier, E.; Blake, C.; Pelecanos, A.; Bauer, J.; Brown, T. Assessing the Concurrent Validity and Interrater Reliability of Patient-Led Screening Using the Malnutrition Screening Tool in the Ambulatory Cancer Care Outpatient Setting. J Acad Nutr Diet 2020, 120, 1210-1215, doi:10.1016/j.jand.2019.10.015.

23. Hsueh, S.W.; Liu, K.H.; Hung, C.Y.; Tsai, C.Y.; Hsu, J.T.; Tsang, N.M.; Hsueh, W.H.; Yang, C.; Chou, W.C. Predicting Postoperative Events in Patients With Gastric Cancer: A Comparison of Five Nutrition Assessment Tools. In vivo (Athens, Greece) 2020, 34, 2803-2809, doi:10.21873/invivo.12106.

24. Huang, D.D.; Cai, H.Y.; Chen, X.Y.; Dong, W.X.; Wangchuk, D.; Yan, J.Y;; Chen, X.L.; Dong, Q.T. Value of Sarcopenia defined by the new EWGSOP2 consensus for the prediction of Postoperative Complications and Long-term Survival after Radical Gastrectomy for Gastric Cancer: A comparison with four common nutritional screening tools. J Cancer 2020, 11, 5852-5860, doi:10.7150/jca.49815.

25. Chen, X.Y.; Zhang, X.Z.; Ma, B.W.; Li, B.; Zhou, D.L.; Liu, Z.C.; Chen, X.L.; Shen, X.; Yu, Z.; Zhuang, C.L. A comparison of four common malnutrition risk screening tools for detecting cachexia in patients with curable gastric cancer. Nutrition (Burbank, Los Angeles County, Calif.) 2020, 70, 110498, doi:10.1016/j.nut.2019.04.009.

26. Lim, H.S.; Lee, B.; Cho, I.; Cho, G.S. Nutritional and Clinical Factors Affecting Weight and Fat-Free Mass Loss after Gastrectomy in Patients with Gastric Cancer. Nutrients 2020, 12, doi:10.3390/nu12071905.

27. Park, J.H.; Kim, E.; Seol, E.M.; Kong, S.H.; Park, D.J.; Yang, H.K.; Choi, J.H.; Park, S.H.; Choe, H.N.; Kweon, M., et al. Prediction Model for Screening Patients at Risk of Malnutrition After Gastric Cancer Surgery. Annals of surgical oncology 2021, 10.1245/s10434-02009559-3, doi:10.1245/s10434-020-09559-3.

28. Zhang, H.; Shang, X.; Ren, P.; Gong, L.; Ahmed, A.; Ma, Z.; Ma, R.; Wu, X.; Xiao, X.; Jiang, H., et al. The predictive value of a preoperative systemic immune-inflammation index and prognostic nutritional index in patients with esophageal squamous cell carcinoma. $J$ Cell Physiol 2019, 234, 1794-1802, doi:10.1002/jcp.27052.

29. Pan, X.; Wang, C.; Li, R.; Su, L.; Zhang, M.; Cai, C.; Liu, S.; Zhang, W.; Song, J.; Hong, J. Applicability of the Nutrition Risk Screening 2002 Combined with a Patient-Generated Subjective Global Assessment in Patients with Nasopharyngeal Carcinoma. Cancer management and research 2020, 12, 8221-8227, doi:10.2147/cmar.S261945.

30. Jingyong Xu; Jian Yang; Weiming Kang; Qian Lu; Zhuming Jiang. Nutrition risk and nutrition risk screening tool Nutrition Risk Screening 2002 clinical application expert consensus (2018 Edition). Chinese Journal of clinical nutrition 2018, 26, $131-135$.

31. Ziyu Li; Chao Yan; Shen Li. Chinese expert consensus on perioperative nutritional therapy for gastric cancer (2019 Edition). Chinese Journal of Practical Surgery 2020, 40, 145-151.

32. Ryu, S.W.; Kim, I.H. Comparison of different nutritional assessments in detecting malnutrition among gastric cancer patients. World journal of gastroenterology 2010, 16, 3310-3317, doi:10.3748/wjg.v16.i26.3310.

33. Maeda, K.; Ishida, Y.; Nonogaki, T.; Mori, N. Reference body mass index values and the prevalence of malnutrition according to the Global Leadership Initiative on Malnutrition criteria. Clinical nutrition (Edinburgh, Scotland) 2020, 39, 180-184, doi:10.1016/j.clnu.2019.01.011.

34. Polit, D.F.; Beck, C.T.; Owen, S.V. Is the CVI an acceptable indicator of content validity? Appraisal and recommendations. Res Nurs Health 2007, 30, 459-467, doi:10.1002/nur.20199.

35. Mokkink, L.B.; Terwee, C.B.; Patrick, D.L.; Alonso, J.; Stratford, P.W.; Knol, D.L.; Bouter, L.M.; de Vet, H.C. The COSMIN study reached international consensus on taxonomy, terminology, and definitions of measurement properties for health-related patient-reported outcomes. J Clin Epidemiol 2010, 63, 737-745, doi:10.1016/j.jclinepi.2010.02.006.

36. Mokkink, L.B.; Terwee, C.B.; Knol, D.L.; Stratford, P.W.; Alonso, J.; Patrick, D.L.; Bouter, L.M.; de Vet, H.C. The COSMIN checklist for evaluating the methodological quality of studies on measurement properties: a clarification of its content. BMC Med Res Methodol 
2010, 10, 22, doi:10.1186/1471-2288-10-22.

37. Hajian-Tilaki, K. Sample size estimation in diagnostic test studies of biomedical informatics. J Biomed Inform 2014, 48, 193-204, doi:10.1016/j.jbi.2014.02.013.

38. Terwee, C.B.; Bot, S.D.; de Boer, M.R.; van der Windt, D.A.; Knol, D.L.; Dekker, J.; Bouter, L.M.; de Vet, H.C. Quality criteria were proposed for measurement properties of health status questionnaires. J Clin Epidemio/ 2007, 60, 34-42, doi:10.1016/j.jclinepi.2006.03.012.

39. Streiner, D.L. Starting at the beginning: an introduction to coefficient alpha and internal consistency. J Pers Assess 2003, 80, 99-103, doi:10.1207/s15327752jpa8001_18.

40. Streiner, D.L. Being inconsistent about consistency: when coefficient alpha does and doesn't matter. J Pers Assess 2003, 80, 217222, doi:10.1207/s15327752jpa8003_01.

41. Manzar, M.D.; Jahrami, H.A.; Bahammam, A.S. Structural validity of the Insomnia Severity Index: A systematic review and metaanalysis. Sleep Med Rev 2021, 60, 101531, doi:10.1016/j.smrv.2021.101531.

42. Eusebi, P. Diagnostic accuracy measures. Cerebrovasc Dis 2013, 36, 267-272, doi:10.1159/000353863.

43. Aoyama, T.; Sato, T.; Hayashi, T.; Yamada, T.; Cho, H.; Ogata, T.; Oba, K.; Yoshikawa, T. Does a laparoscopic approach attenuate the body weight loss and lean body mass loss observed in open distal gastrectomy for gastric cancer? a single-institution exploratory analysis of the JCOG 0912 phase III trial. Gastric cancer: official journal of the International Gastric Cancer Association and the Japanese Gastric Cancer Association 2018, 21, 345-352, doi:10.1007/s10120-017-0735-4.

44. Mokkink, L.B.; Terwee, C.B.; Patrick, D.L.; Alonso, J.; Stratford, P.W.; Knol, D.L.; Bouter, L.M.; de Vet, H.C. The COSMIN checklist for assessing the methodological quality of studies on measurement properties of health status measurement instruments: an international Delphi study. Qual Life Res 2010, 19, 539-549, doi:10.1007/s11136-010-9606-8.

45. Rattray, J.; Jones, M.C. Essential elements of questionnaire design and development. Journal of clinical nursing 2007, 16, 234-243, doi:10.1111/j.1365-2702.2006.01573.x.

46. Crossley, K.M.; Macri, E.M.; Cowan, S.M.; Collins, N.J.; Roos, E.M. The patellofemoral pain and osteoarthritis subscale of the KOOS (KOOS-PF): development and validation using the COSMIN checklist. Br J Sports Med 2018, 52, 1130-1136, doi:10.1136/bjsports2016-096776.

47. Mohamad, H.; Ntessalen, M.; Craig, L.C.A.; Clark, J.; Fielding, S.; N'Dow, J.; Heys, S.D.; McNeill, G. A self-help diet and physical activity intervention with dietetic support for weight management in men treated for prostate cancer: pilot study of the Prostate Cancer Weight Management (PRO-MAN) randomised controlled trial. The British journal of nutrition 2019, 122, 592-600, doi:10.1017/s0007114519001090.

48. Kim, H.J.; Kim, H.S. Effects of a web-based expert support self-management program (WEST) for women with breast cancer: A randomized controlled trial. J Telemed Telecare 2020, 26, 433-442, doi:10.1177/1357633×19850386.

\section{Table}

Table 1 Inclusion and exclusion criteria

\begin{tabular}{lll}
\hline & Inclusion criteria & Exclusion criteria \\
\hline Population & Patients with gastric cancer and gastrectomy. & Patients with complications after gastric cancer surgery. \\
\hline Exposure & Nutritional supplement. & Herbal supplements \\
& & Follow-up less than one month.
\end{tabular}

Outcomes $\quad$ BMI ; Weight loss; Reduced muscle mass; Reduced
food intake or assimilation.

$\begin{array}{ll}\text { Study design } & \text { Clinical studies (controlled } \\ & \text { and uncontrolled clinical trials, observational studies). }\end{array}$

Publication type Full text articles.

Language English,Chinese.

\section{Figures}



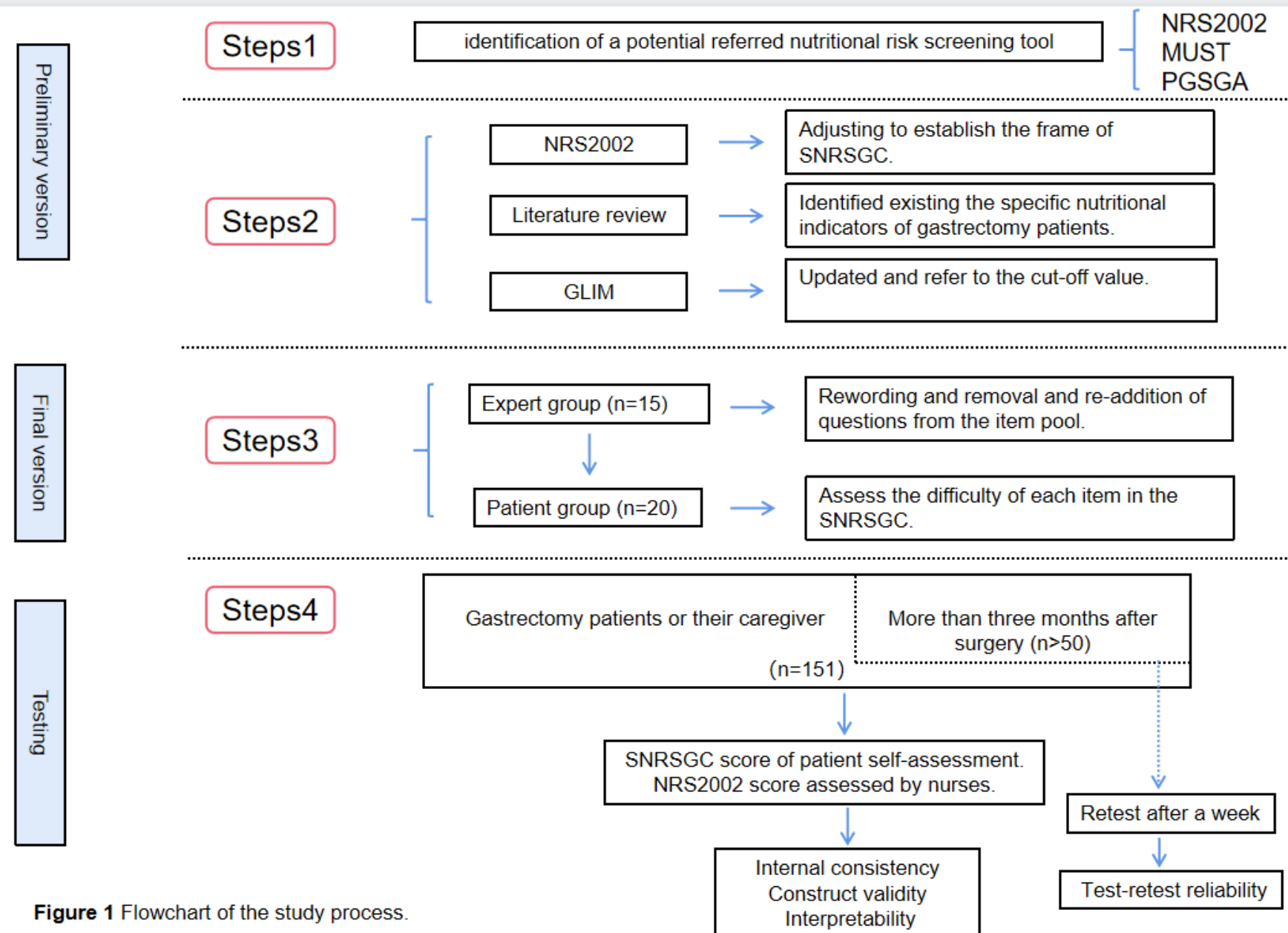

Figure 1 Flowchart of the study process

Construct validity

Test-retest reliability

\section{Figure 1}

See image above for figure legend. 